\title{
Carotid artery wall segmentation by coupled surface graph cuts
}

Arias, Andres; Petersen, Jens; van Engelen, Arna; Tang, Hui; Selwaness, Mariana;

Witteman, Jacqueline C. M.; van der Lugt, Aad; Niessen, Wiro; de Bruijne, Marleen

Published in:

Medical Computer Vision. Recognition techniques and applications in medical imaging

DOI:

10.1007/978-3-642-36620-8_5

Publication date:

2013

Document version

Peer reviewed version

Citation for published version (APA):

Arias, A., Petersen, J., van Engelen, A., Tang, H., Selwaness, M., Witteman, J. C. M., van der Lugt, A., Niessen, W., \& de Bruijne, M. (2013). Carotid artery wall segmentation by coupled surface graph cuts. In B. H. Menze, G. Langs, L. Lu, A. Montillo, Z. Tu, \& A. Criminisi (Eds.), Medical Computer Vision. Recognition techniques and applications in medical imaging: Second International MICCAI Workshop, MCV 2012, Nice, France, Revised Selected Papers (pp. 38-47). Springer. Lecture notes in computer science Vol. 7766 https://doi.org/10.1007/9783-642-36620-8_5 


\title{
Carotid artery wall segmentation by coupled surface graph cuts
}

\author{
Andres Arias ${ }^{1}$, Jens Petersen ${ }^{2}$, Arna van Engelen ${ }^{1}$, Hui Tang ${ }^{1,3}$, Mariana \\ Selwaness $^{4,5,6}$, Jacqueline C.M. Witteman ${ }^{5}$, Aad van der Lugt ${ }^{4}$, Wiro \\ Niessen $^{1,3}$, and Marleen de Bruijne ${ }^{1,2}$ \\ 1 Biomedical Imaging Group Rotterdam, Departments of Radiology and Medical \\ Informatics, Erasmus MC, Rotterdam, The Netherlands \\ ${ }^{2}$ Image Group, Department of Computer Science, University of Copenhagen, \\ Denmark \\ 3 Faculty of Applied Sciences, Department of Imaging Science and Technology, Delft \\ University of Technology, The Netherlands \\ ${ }^{4}$ Department of Radiology, Erasmus MC, Rotterdam, The Netherlands \\ 5 Department of Epidemiology, Erasmus MC, Rotterdam, The Netherlands \\ ${ }^{6}$ Department of Biomedical Engineering, Erasmus MC, Rotterdam, The Netherlands
}

\begin{abstract}
We present a three-dimensional coupled surface graph cut algorithm for carotid wall segmentation from Magnetic Resonance Imaging (MRI). Using cost functions that highlight both inner and outer vessel wall borders, the method combines the search for both borders into a single graph cut. Our approach requires little user interaction and can robustly segment the bifurcation section of the carotid artery. Experiments on 32 carotid arteries from 16 patients show good agreement between manual segmentation performed by an expert and our method. The mean relative area of overlap is more than $85 \%$ for the lumen and outer vessel wall. In addition, differences in measured wall thickness with respect to the manual annotations were smaller than the image pixel size.
\end{abstract}

Keywords: Carotid artery, flow lines, graph, segmentation.

\section{Introduction}

Atherosclerosis is one of the primary causes of death in the world [10]. Atherosclerotic plaques in the carotid arteries cause lumen narrowing. This may lead to plaque rupture, which can cause a stroke or Transient Ischemic Attack (TIA). Therefore, the early detection and quantification of plaque is important. To accurately quantify the extent of carotid atherosclerosis it is essential to segment the vessel wall. As manual segmentation is highly time consuming and subject to observer variability, therefore, automated techniques are needed.

Although most work on automated segmentation of blood vessels has focused on segmenting the vessel lumen only, several automatic and semi-automatic methods have been proposed in the past for segmenting the outer vessel wall. Active Shape Models (ASMs) have been used for detecting the outer vessel 
wall of the abdominal aorta in CTA scans [2]. These ASMs utilize a statistical model of shape and boundary grey level appearance to restrict the search space to anatomically reasonable solutions. To segment the carotid arteries in MRI, gradient-based ellipse fitting combined with fuzzy clustering [1] and Closed Contour Snakes (CCS) [14] have been proposed. A drawback of both these methods is that user interaction is required for each image slice. More recently, van 't Klooster et al. [7] proposed a three-dimensional (3D) deformable vessel model, in which a vessel is modeled using a 3D cylindrical surface that can be modified by moving control points located at the model surface. Good results were achieved on MR images of the carotids. This method can however segment only a single, non-bifurcating vessel and will therefore not give reliable results in the bifurcation region. Better segmentation results may be achieved if both walls are estimated jointly across the bifurcation and if local image information is combined with a globally optimal solution.

Global optimality can be guaranteed with graph based methods, and recently these have been used for vessel segmentation with promising results $[8,9,4,12]$. Surface based graph methods such as $[8,9,12]$ as opposed to voxel based [4] make it possible to enforce topology constraints as well as penalizing smoothness without biasing the solution towards smaller surfaces. Using these methods the problem has to be transformed from image space to a discretized graph space defined by a set of columns. Each column is associated with a point on the sought surface and represents the set of possible solutions, or positions, it can take. The suitability of the graph space depends on how well the graph columns cross the sought surface [9]. Xu et al. [12] oriented the graph columns in the normal direction of the centerline of the vessels, but this leads to long columns and thus in-efficiency if the sought surface is far from the centerline. Moreover, straight columns intersect in regions with curvature leading to possibly self-intersecting surfaces [9].

We propose to use a 3D coupled surface graph cut algorithm for carotid artery wall segmentation from MRI images. Similar to Petersen et al. [9] who applied such a technique for segmenting airway trees, we define the graph columns based on flow lines traced from a coarse initial segmentation, which, because they are non-intersecting, allow accurate segmentation across high curvature areas such as those found in the carotid bifurcation region. Moreover as the surfaces are estimated jointly, the proposed method can use information from both surfaces locally and globally to reach an optimal solution.

\section{Method}

\subsection{Initial Segmentation}

An initial segmentation of the lumen was obtained using the method proposed by Tang et al. [11]. In this method first the lumen centerlines are determined by finding a minimum cost path between three user-defined seed points in the common, internal, and external carotid arteries. Then refined iteratively by doing a minimum cost path in a curved multi-planary reformatting. Subsequently, the 
lumen is segmented using a levelset method, which is initialized by the extracted centerlines and steered by the MR intensities.

\subsection{Graph Construction}

First, to obtain the graph columns the initial segmentation was converted to a mesh. We located graph vertices at the center of each surface face. This set of vertices is denoted by $V_{B}$.

Flow Lines The graph columns are traced from $V_{B}$, and follow the direction of flow lines of the gradient vector field of the smoothed segmentation. An example of columns traced along flow lines is depicted in figure 1. The flow lines define a set of positions in the Euclidean space which are oriented along a gradient vector field at each point. If this gradient vector field is defined in terms of a scalar potential field $\phi$, it will follow the direction with the greatest rate of change of this potential. We define the scalar field $\phi$ by the convolution of the initial segmentation with a Gaussian kernel $G_{\sigma}$ as:

$$
\phi(\boldsymbol{x})=\int Q(\hat{\boldsymbol{x}}) G_{\sigma}(\hat{\boldsymbol{x}}-\boldsymbol{x}) d \hat{\boldsymbol{x}}
$$

where $Q: \mathbb{R}^{3} \rightarrow \mathbb{Z}$ is the initial lumen segmentation represented by a binary scalar field. Flow lines traced along the gradient of $\phi$ have the properties of being smooth and non-self-intersecting and the surfaces are thus non-self-intersecting [9]. These properties are also depicted in figure 1.

To compute the flow lines, we recall that these are oriented along the gradient of the scalar field $\phi$ and cross the initial surface. Therefore, the parametric flow lines $f: \mathbb{R} \rightarrow \mathbb{R}^{3}$ that cross each vertex of the initial surface mesh $\boldsymbol{i}_{0} \in V_{B}$ can be computed by solving the following differential equation:

$$
\frac{\partial \boldsymbol{f}}{\partial t}(t)=\nabla \phi(\boldsymbol{f}(t))
$$

with initial value given by $\boldsymbol{f}(0)=\boldsymbol{i}_{0}$.

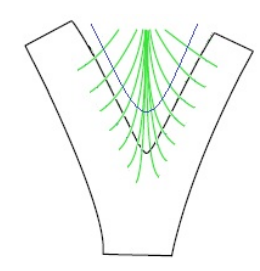

Fig. 1. Graph columns based on flow lines (green) traced from an initial segmentation (black), which are crossing the sought surface (blue). 
Solving equation (2) for all vertices on the initial surface mesh $V_{B}$ leads to all graph columns, where inner and outer graph columns are represented by the same flow lines. We use the Runge-Kutta-Fehlberg method to approximate the solution of these differential equations [3]. The solution of $\boldsymbol{f}(t)$ is approximated at regular intervals $\delta$ along the line. This defines the positions of the other graph vertices. The length of the columns vary depending on the point where the gradient of the scalar field $\phi$ flattens.

Graph construction and optimization To construct the coupled surface graph $G=(V, E)$ with vertices $V$ and edges $E$, we define the set of vertices in a column by $V_{i}$ with $i \in V_{B}$. Therefore, the complete set of vertices $V$ is defined by:

$$
V=\bigcup_{i \in V_{B}, m \in M} V_{i}^{m} \cup\{s, t\}
$$

Here $M$ represents the surfaces to find (in our specific case there are two surfaces: inner and outer surface), $s$ and $t$ denote the source and sink vertices respectively. Moreover, given that the inner and outer columns are the same, we have $\forall_{m \in M} V_{\boldsymbol{i}}^{m}=V_{\boldsymbol{i}}$.

The edge set $E$ of the coupled surface graph $G$ consists of intra-column edges $E_{\text {intra }}$ and edges between columns $E_{\text {inter }}$. For the intra-column edges $E_{\text {intra }}$, we define direct edges connecting from each vertex to the next outward following vertex in the same column. We assign edges from the source vertex $s$ to all inner most vertices in the graph, and from the outer most vertices to the sink vertex $t$. Topology preserving edges in the opposite direction with infinity capacity ensure that a minimum cut can only cut each column once. In addition, we define a cost function $w^{m}\left(i_{k}^{m}\right)>0$ to these edges, mapping a vertex with index $k$ in column $V_{\boldsymbol{i}}$ to the inverse likelihood representation that it is part of surface $m$. An example of the intra-column edges and their respective costs is shown in figure 2(a) (for simplicity we do not show the infinity capacity edges).

Selecting a vertex for each column indicates a possible solution for all $M$ surfaces. Therefore, a cut that separates the graph in two parts: sink and source, represents a solution to the segmentation problem. The main aim is then to find a cut that minimizes the cost of the edges that are being cut as depicted in figure 2(b). There are several approaches to solve this optimization problem. We used a min-cut/max-flow algorithm described in [13] to find the minimum cut.

Computing the minimum cut without considering any interaction between columns may lead to irregular surfaces and/or un-realistic relations between surfaces such as borders that are too far from each other, or an outer surface that is inside the inner surface. In order to deal with these problems, we include smoothing penalty edges connecting vertices in columns belonging to the same surface, separation penalty edges and separation constraint edges that connect vertices from columns of different surfaces. These represent the edges between columns $E_{\text {inter }}$. 
To ensure smooth surfaces, we linearly penalize the distance in a cut between consecutive columns of the same surface. To do this, we assign edges with the same capacity $p$ between vertices at the same column level. When the length of two consecutive columns are different, the remaining vertices at the inner most part of the column are connected to the source vertex, and the remaining vertices at the outer most part of the column are connected to the sink. If these edges coincide with the intra-column edges, only one edge is assigned and the capacities are added. An example of these smoothing penalty edges is shown in figure 2(c). Using these edges we obtain a linear penalty function of the form $\psi(x)=p x$, where $x$ represents the vertex index difference. In a similar way, the separation between surfaces is penalized by assigning edges with capacity $q$ to edges between vertices of columns lying at the same flow line but belonging to different surfaces. In addition, to avoid solutions where parts of the outer surfaces are inside the inner surface, we assign constraint edges with infinite capacity at the same location of the separation edges but pointing from the inner column to the outer column. These edges make an optimal cut where outer surfaces are inside an inner surface unfeasible. An example of these separation penalty edges and separation constraint edges is shown in figure $2(\mathrm{~d})$.

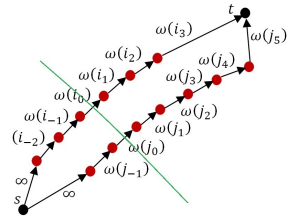

(a)

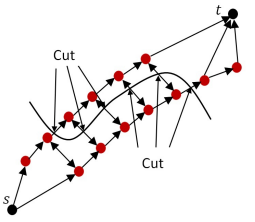

(b)

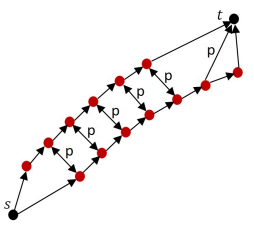

(c)

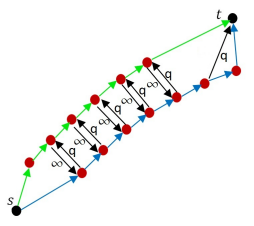

(d)

Fig. 2. Examples of intra and inter column edges and a graph cut. In figure 2(a) the intra-column edges, the initial segmentation (green), and the associated cost to each edge are depicted. An example of a graph cut is depicted in figure 2(b), indicating which edges are part of the cut. Figure 2(c) shows the smooth penalty edges which connect vertices from neighbor columns of the same surface. Finally, the separation penalty edges and separation constraint edges are depicted in figure $2(\mathrm{~d})$. These edges connect vertices from columns of different surfaces lying at the same flow line (green: inner column, blue: outer column).

\subsection{Cost Functions}

For the intra-column edges, we define a cost function $w^{m}\left(i_{k}^{m}\right)>0$. The outcome of this function is a representation of the inverse likelihood that the vertex $i_{k}^{m}$ associated to the edge $i_{k}^{m} \rightarrow i_{k+1}^{m}$ is part of surface $m$. In the case of the carotid walls in our MR images, the graph columns will start inside the lumen area, which looks dark in the image, move through the carotid wall where the voxels are normally brighter, and finally end up out of the carotid wall where the image 
is darker compared to the wall intensity. We therefore define a cost function for the inner wall $w^{i}$ which is low for strong dark-to-bright edges, and a cost function for the outer wall $w^{o}$ which is low for strong bright-to-dark edges. There are several methods in the literature for finding the edges using derivatives $[8,9]$. A common approach is to use the fact that the first and second order derivative edge detectors place their maximums on different sides of the sought edge $[8,9]$. Weightings of these derivatives can therefore be used to adjust the position of the found edge, in order to match some form of ground truth data. We use a similar approach to Petersen et al. [9] to define the cost functions. First, we define the functions $C^{i}: \mathbb{R} \rightarrow \mathbb{R}$ and $C^{o}: \mathbb{R} \rightarrow \mathbb{R}$ that highlight the inner and outer walls. These use a linear combination of the first and second order derivatives of the intensity along the columns:

$$
\begin{gathered}
C^{i}(t)=\gamma^{i} \frac{\partial P}{\partial t}(t)+\left(1-\left|\gamma^{i}\right|\right) P(t), \\
C^{o}(t)=\gamma^{o} \frac{\partial N}{\partial t}(t)+\left(1-\left|\gamma^{o}\right|\right)(-N(t)),
\end{gathered}
$$

where $\gamma^{i}, \gamma^{o} \in[-1,1]$ are weighting values, and $P$ and $N$ the positive and negative parts of the first order derivative respectively. These derivatives are computed using central differences from cubic interpolated values. Subsequently, we invert and normalize $C^{i}$ and $C^{o}$ in order to get a representation of the wall inverse likelihood given by $w^{i}$ and $w^{o}$.

\section{Experiments and Results}

\subsection{Data}

Proton Density Weighted Black-Blood MRI (BBMRI) and Proton Density Weighted Echo Planar MRI (EPIMRI) images were obtained from 26 subjects that were randomly selected from the Rotterdam study [5]. Briefly, BBMRI images were acquired using an in-plane pixel size of $0.51 \mathrm{~mm} \times 0.51 \mathrm{~mm}$, and 0.9 $\mathrm{mm}$ slice thickness. The EPIMRI images had a slice thickness of $1.2 \mathrm{~mm}$ and same in-plane resolution as BBMRI images. Affine registration using mutual information from EPIMRI to BBMRI was performed using Elastix [6]. In addition, to train and evaluate our method, we used manually annotated cross-sectional images with a resolution of $0.05 \mathrm{~mm} \times 0.05 \mathrm{~mm}$ extracted at random positions perpendicular to the carotid artery center-lines. The manual annotations of the inner and outer carotid walls were drawn by an expert on the BBMRI images. Six manually annotated cross sections were extracted from each carotid artery.

\subsection{Evaluation}

We evaluate the correctness of our method based on several quantitative parameters. We use the relative area of overlap (also called average Dice's coefficient). In addition, we also compared the wall thickness [7] and cross-sectional areas [1] obtained from the manual and automatic segmentations. 


\subsection{Graph parameters tuning}

The proposed method method described in this paper has several parameters: inner and outer smoothness penalties $p^{i}$ and $p^{o}$, separation penalties $q$, inner and outer cost function derivative weightings $\gamma^{i}$ and $\gamma^{o}$, the intervals for sampling the flow lines to define the positions of the vertices $\delta$, and the standard deviation of the Gaussian kernel $\sigma$. We used the carotid arteries of ten patients randomly selected to search for the optimal values for these parameters on each image sequence (BBMRI and EPIMRI). The optimal values were obtained by searching the parameter space on the training data-set using an iterative binary search algorithm [9]. In this algorithm, manually annotated cross-sections and automatic segmented cross-sections are compared based on the relative area of overlap. The set of parameters that generated the highest overlap was selected. To reduce the searching time of the parameter optimization algorithm, we fixed the column sampling interval $\delta$ to $0.35 \mathrm{~mm}$.

\subsection{Segmentation Results}

Thirty two carotid arteries of 16 patients not included in the training set were used for the evaluation. Table 1 gives the average relative area of overlap for inner $\varphi^{i}$ and outer vessel surface $\varphi^{o}$ on this testing data set. In addition, table 1 describes the mean signed and mean absolute difference between wall thickness measured by the manual annotation and by the automatic segmentations (WTD) in BBMRI and EPIMRI. Notice that these values are smaller than the image in plane pixel size $(0.51 \mathrm{~mm})$. We observed good segmentation overlap for both sequences with a slightly higher overlap for BBMRI images. Using EPIMRI images we obtained lower WTD. In the table is also shown the mean cross-section lumen area difference (LAD), and mean cross-section outer vessel area difference (OVAD). $P$-values of the paired $t$-test including $95 \%$ of confidence intervals are also given in the table. Figure 3(a) shows examples of the automatic segmentation results using BBMRI and EPIMRI images together with the overlay to the manual annotations. Results in the bifurcation section are depicted in figure 3(b). Moreover, we do not show overlay images because manual annotations are not available for these slices. Figure 3(c) shows a 3D representation of the automatic segmentation.

Table 1. Relative area of overlap, WTD, LAD, and OVAD for both sequences (in parentheses are shown the mean absolute and $P$-values).

\begin{tabular}{|c|c|c|}
\hline & BBMRI & EPIMRI \\
\hline$\varphi^{i}$ & $85.2 \% \pm 1.6 \%$ & $83.9 \% \pm 5 \%$ \\
\hline$\varphi^{o}$ & $85.6 \% \pm 2.7 \%$ & $84.1 \% \pm 6 \%$ \\
\hline WTD $(\mathrm{mm})$ & $-0.25 \pm 0.24(0.28 \pm 0.19 ; p<0.001)$ & $0.04 \pm 0.23(0.17 \pm 0.15 ; p=0.5)$ \\
\hline $\operatorname{LAD}\left(\mathrm{mm}^{2}\right)$ & $2.7 \pm 2.1(3.0 \pm 1.7 ; p<0.001)$ & $-0.07 \pm 3.29(2.3 \pm 2.21 ; p=0.9)$ \\
\hline OVAD $\left(\mathrm{mm}^{2}\right)$ & $-0.3 \pm 1.1(0.92 \pm 0.8 ; p=0.3)$ & $-0.3 \pm 1.05(0.76 \pm 0.75 ; p=0.25)$ \\
\hline
\end{tabular}


Bland-Altman analysis for the mean wall thickness, mean cross-section lumen area, and mean cross-section outer vessel area for the 16 patient data sets is shown in figure 4 . From the figure is observed a good agreement between automatic and manual area measurements for lumen and outer vessel wall. Pearson correlation coefficients were 0.95 and 0.98 respectively for BBMRI, and 0.87 and 0.99 for EPIMRI.

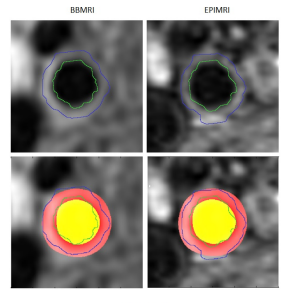

(a)

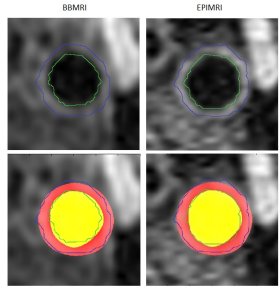

)

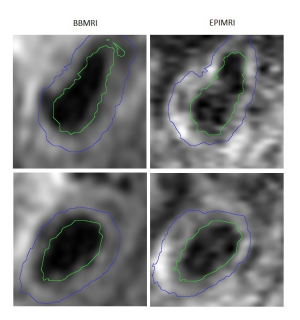

(b)

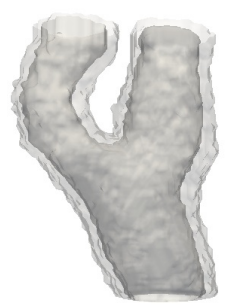

(c)

Fig. 3. Automatic segmentation results using the proposed method. In figure 3(a), two example of automated segmented cross-section in BBMRI and EPIMRI are depicted (top). The automatic segmentation is represented by green (inner wall) and blue (outer wall) lines. The overlay of the automatic segmentation to the manual annotations (yellow: lumen, red: vessel wall) is also depicted in figure 3(a) (bottom). Figure 3(b) shows two example (top and bottom images) of automated segmented cross-sections in the bifurcation section. Finally, figure 3(c) shows a 3D representation of the automatic segmentation of the complete carotid artery in an image (darker gray: lumen, bright gray: outer wall).

\section{Discussion and Conclusion}

In this paper, we presented a new 3D method for carotid wall segmentation in MRI. Results show a good agreement between manual segmentation performed by an expert and our method. The mean relative area of overlap was about $84 \%$ and $85 \%$ for EPIMRI and BBMRI respectively. Our results are comparable to or slightly better than those reported in the literature. Van 't Klooster et al. [7] reported a WTD of $0.12 \mathrm{~mm} \pm 0.21 \mathrm{~mm}$. We found a somewhat lower mean WTD with a similar variance $0.04 \mathrm{~mm} \pm 0.23 \mathrm{~mm}$ using the EPIMRI sequence. In addition, their method only analyzes the common carotid artery and not the bifurcation. This section may represent the most difficult section to segment. In contrast, we analyze the complete carotid artery.

Adam et al. [1] use similar in-plane resolution images of 17 patients. They reported a LAD of $-2.19 \mathrm{~mm}^{2} \pm 5.21 \mathrm{~mm}^{2}$ and an OVAD of $-5.56 \mathrm{~mm}^{2} \pm 19.55 \mathrm{~mm}^{2}$ with correlation coefficients of 0.92 and 0.91 respectively. Yuan et al. [14] reported a LAD of $1.05 \mathrm{~mm}^{2} \pm 2.26 \mathrm{~mm}^{2}$ and an OVAD of $1.36 \mathrm{~mm}^{2} \pm 3.46 \mathrm{~mm}^{2}$ 


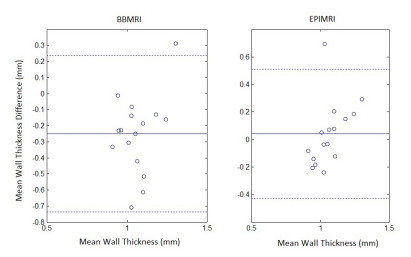

(a)

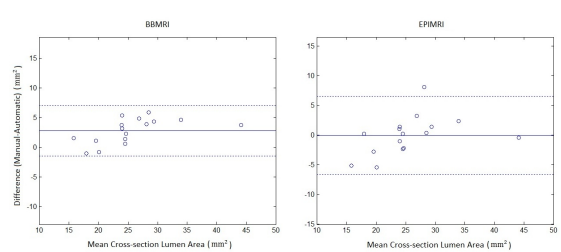

(b)

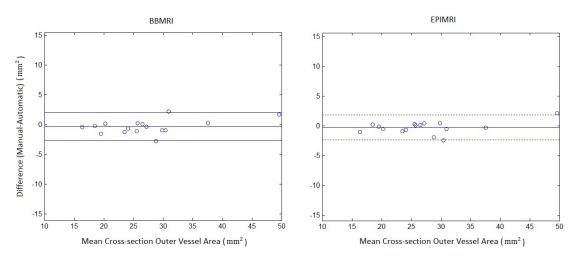

(c)

Fig. 4. Bland-Altman plots comparing manual annotations and automatic segmentation for both sequences BBMRI and EPIMRI. Figure 4(a) depicts the comparison of the mean wall thickness. Figure 4(b) and figure 4(c) show a comparison of the mean lumen area and outer vessel area respectively.

on five patients, and focus on the internal carotid artery. We reported in general better results on our data compared to these two methods (see table 1). Furthermore, these two methods require a large amount of user interaction. In contrast, our method only requires the location of three seed points for obtaining the initial segmentation.

A potential drawback of our approach is that it relies on an initial lumen segmentation. Although this segmentation does not need to be very accurate, the smoothness constraints are most effective if the shape of the initial segmentation is similar to shape of the true vessel surfaces. Another potential source of errors in our method is related to registration errors of the EPIMRI images. In addition, disagreements between manual and automatic segmentation may be generated by the inherit subjectivity of manually draw the vessels contours. Overall, segmentation results were best for the sequence in which the manual annotations were performed, the BBMRI. However, the EPIMRI images have better wall contrast, which generates better results in some images compared to the results obtained by BBMRI. Therefore, we expect that combining information from both image types in the cost function can still improve upon the results presented here.

To conclude, we propose a graph-based method for segmenting the carotid artery wall that shows good agreement with manual segmentations. In contrast to previous approaches, our method can segment the bifurcation section, which may be the most clinically relevant area to assess. 


\section{References}

1. Adame, I.M., van der Geest, R.J., Wasserman, B.A., Mohamed, M.A., Reiber, J.H.C., Lelieveldt, B.P.F.: Automatic segmentation and plaque characterization in atherosclerotic carotid artery $\mathrm{mr}$ images. Magnetic Resonance Materials in Physics, Biology and Medicine 16, 227-234 (2004)

2. de Bruijne, M., van Ginneken, B., Viergever, M.A., Niessen, W.J.: Adapting active shape models for $3 \mathrm{~d}$ segmentation of tubular structures in medical images. In: in medical images," in Information Processing in Medical Imaging. pp. 136-147. Springer (2003)

3. Butcher, J.: Numerical Methods for Ordinary Differential Equations. Wiley (2008)

4. Freiman, M., Frank, J., Weizman, L., Nammer, E., Shilon, O., Joskowicz, L., Sosna, J.: Nearly automatic vessels segmentation using graph-based energy minimization. The MIDAS Journal (2009)

5. Hofman, A., Duijn, C.v., Franco, O., Ikram, M., Janssen, H., Klaver, C., Kuipers, E., Nijsten, T., Stricker, B., Tiemeier, H., Uitterlinden, A., Vernooij, M., Witteman, J.: The rotterdam study: 2012 objectives and design update. European Journal of Epidemiology 2011, 1-30 (August 2011)

6. Klein, S., Staring, M., Murphy, K., Viergever, M., Pluim, J.: elastix: a toolbox for intensity-based medical image registration. IEEE Transactions on Medical Imaging 29(1), $196-205$ (2010)

7. van 't Klooster, R., de Koning, P.J., Dehnavi, R.A., Tamsma, J.T., de Roos, A., Reiber, J.H., van der Geest, R.J.: Automatic lumen and outer wall segmentation of the carotid artery using deformable three-dimensional models in mr angiography and vessel wall images. Journal of Magnetic Resonance Imaging 35(1), 156-165 (2012)

8. Li, K., Wu, X., Chen, D.Z., Sonka, M.: Optimal surface segmentation in volumetric images - a graph-theoretic approach. IEEE TRANS. PATTERN ANAL. MACHINE INTELL 28(1), 119-134 (2006)

9. Petersen, J., Nielsen, M., Lo, P., Saghir, Z., Dirksen, A., De Bruijne, M.: Optimal graph based segmentation using flow lines with application to airway wall segmentation. In: Proceedings of the 22nd international conference on Information processing in medical imaging. pp. 49-60. IPMI'11, Springer-Verlag, Berlin, Heidelberg (2011)

10. Ross, R.: Atherosclerosis - an inflammatory disease. New England Journal of Medicine 340(2), 115-126 (1999)

11. Tang, H., van Walsum, T., van Onkelen, R.S., Hameeteman, K., Klein, S., Schaap, M., Bouwhuijsen, Q.J.B., Witteman, J., van der Lugt, A., van Vliet, L.J., Niessen, W.: Semiautomatic carotid lumen segmentation for quantification of lumen geometry in multispectral mri. Medical Image Analysis (May 2012)

12. Xu, X., Niemeijer, M., Song, Q., Garvin, M.K., Reinhardt, J.M., Abramoff, M.D.: Retinal vessel width measurements based on a graph-theoretic method, p. 641-644. IEEE (2011)

13. Y., B.: An experimental comparison of min-cut/max-flow algorithms for energy minimization in vision. IEEE Transactions on Pattern Analysis and Machine Intelligence 26(9), 1124-1137 (2004)

14. Yuan, C., Lin, E., Millard, J., Hwang, J.: Closed contour edge detection of blood vessel lumen and outer wall boundaries in black-blood $\mathrm{mr}$ images. Magnetic Resonance Imaging 17(2), 257 - 266 (1999) 